\title{
Efficacy, Safety and Affordability Disparities of Gastrointestinal Cancer Changing Plans of Treatment of Cancer Patients
}

\author{
Rasha Aboelhassan ${ }^{1 *}$, SohaAli ${ }^{2}$, Mohamed Hassan Ali ${ }^{3}$ and Noura Anwar Abdel-Fatah ${ }^{4}$ \\ ${ }^{1}$ Nasser Institute for Research and Treatment, Adult Oncology Unit, Cairo, Egypt \\ ${ }^{2}$ DataClin Research Organization, Cairo, Egypt \\ ${ }^{3}$ Nasser Institute for Research and Treatment, Clinical Pharmacy Unit, Cairo, Egypt \\ ${ }^{4}$ Graduate School of Statistical Research Cairo University, Cairo, Egypt
}

*Corresponding author: Dr. Rasha Aboelhassan, Nasser Institute for Research and Treatment, Adult Oncology Unit, Cairo, Egypt; E-Mail: elsayedonco@hotmail.com Received: January 03, 2022; Accepted: January 10, 2022; Published: January 11, 2022

\begin{abstract}
Purpose: With daily increase of GIT cancer incidence, there is more discovery of new regimens, raising the question: how much can cancer patient afford effective safe treatment?

Methods: Safety and efficacy grades version 2021 NCCN evidence-based blocks are used in this research, Egyptian population divided according to income in to 5 categories: Poorest (4860), poor (8460), middle (22800), rich (41100), richest (66583). We considered a medicine as affordable if $20 \%$ or less of monthly income is needed to cover monthly need of medicine. Affordability divided to 5 grades according to percentage of income needed to cover treatment: very inexpensive: $\leq 20 \%$, inexpensive: $20-40 \%$, moderately expensive $40-60 \%$, expensive $60-80 \%$, awfully expensive $\geq 80 \%$. Binary logistic regression model was performed to assess affordability of cancer treatments in different cancer types using efficacy of regimen, safety of regimen, line of therapy, site of treatment and income class as predictors.
\end{abstract}

Results: Most GIT regimens are moderately effective 223 (51.6), while most regimens 277 (64.1) are mildly toxic. Minimally effective regimens increase the chance of being affordable by 15 times if compared to highly effective $\mathrm{P}<0.0001$, moderately effective increase the chance of affordability by 3 times if compared to highly effective $\mathrm{P}<0.0001$. Mildly toxic regimen increases the chance of affordability by 3 times if compared to occasionally toxic treatment. $\mathrm{P}=0.002$. Adjuvant regimens have increased chance of affordability by 17 times if compared to second line.

Conclusion: GIT cancer patients have more treatment affordability for neo-adjuvant/adjuvant than other regimens first-line therapy is more affordable than other regimens for stage IV disease, highly effective regimens have the low affordability while mildly toxic regimens have more chance of affordability than other regimens with different safety categories.

\section{Introduction}

With the increase of gastrointestinal cancer global burden [1], there is also increase in number of approved drugs for cancer treatment, on the other hand, there is rapid raise of cancer therapy prices all over the world, with associated decrease cancer treatment affordability between cancer patients [2].

The decision of cancer treatment protocol by any committee depends on many factors, not only magnitude of patient benefit and safety of treatment but also affordability and access of treatment for patients [3].

Value based pricing of cancer treatment is one of the most common and important tools for reducing cancer medicine, by these tools we consider efficacy, toxicity, quality-of-life before pricing certain medication for cancer treatment [4]. National cancer comprehensive network NCCN blocks, European society of medica oncologymagnitude of clinical benefit ESMO-MCB and American society of clinical oncology value framework AVF are different stakeholders' frameworks to help assess the value of oncology regimens [5,6].

Oncologists have different perspectives for affordability of cancer therapy in different regions, which result in different cost-efficacy of treatment [7]. Also, there is certain different relation between affordability and efficacy of different cancer treatment in different countries [8].

This article will address relation between affordability, efficacy, safety of GIT cancer therapy in EGYPT.

\section{Methods}

Safety and efficacy grades version 2021 NCCN evidence-based blocks are used as reference for safety and efficacy for treatment protocols.

Egyptian populations were divided according to average monthly income in to 5 categories by EGP: Poorest (4860), poor (8460), middle (22800), rich (41100), richest (66583). 
We considered a medicine as affordable if $20 \%$ or less of monthly income is needed to cover monthly need of medicine.

Affordability divided to 5 grades according to percentage of income needed to cover treatment: very inexpensive: $\leq 20 \%$, inexpensive: $20-40 \%$, moderately expensive $40-60 \%$, expensive $60-80 \%$, awfully expensive $\geq 80 \%[9]$.

Local essential drug list and local drug pricing index are used as reference for cancer pricing of whole regimens with added pricing of supportive care and hospital admission.

Binary logistic regression model was performed to assess affordability of cancer treatments in different cancer types using efficacy of regimen, safety of regimen, line of therapy, site of treatment and income class as predictors and used to investigate the relation between efficacy and safety of regimens in different tumor sites.

\section{Results}

\section{Numbers of Treatment Protocols for Each Tumor Site}

A total of 432 treatment regimens from NCCN blocks N (\%): Colon 166 (38.4), esophageal 88 (20.4), gastric 80 (18.5), pancreatic 70 (16.2) and HCC 28 (6.5) (Figure 1).

In colon cancer, the highest number of treatment protocols are in second line setting 41 (24.7), while protocols for adjuvant, first line, neoadjuvant, subsequent therapy and third line therapy, and primary treatment are showing the following $\mathrm{N}$ (\%): 24 (14.5), 27 (16.3), 14 (8.4), 21 (12.7), 22 (13.3), 17 (10.2) respectively.
For esophageal and esophagogastric junction cancer, the number first line therapy for metastatic disease is the highest 39 (44.3), and third line/subsequent lines are the lowest 2 (2.3).

For gastric cancer first line for metastatic disease are the highest 36 (45\%) and there is only one third line.

For HCC, most treatment protocols are for first line stage IV disease 18 (64.3), and for pancreatic adenocarcinoma most protocols are for first line 33 (47.1) (Table 1 and Figure 1).

\section{Efficacy of Treatment Regimens}

Most of GIT cancer regimens present in NCCN blocks, are moderately effective 223 (51.6), while none of them are highly effective, minimally effective regimens 60 (13.9), very effective regimens 149 (34.5).

While regimens for gastric cancer are very effective 39 (48.8), pancreatic adenocarcinoma accounts for the highest number of minimally effective regimens 22 (31.4).

\section{Safety of Treatment Regimens}

None of treatment regimens for GIT cancers has no toxicity, most of them are mildly toxic 277 (64.1).

Colon cancer account for the only highly toxic regimens 2 (1.2) in GIT cancer while HCC account for the highest number of mildly toxic regimens 24 (85\%) (Tables 1, 2, Figures 2 and 3).

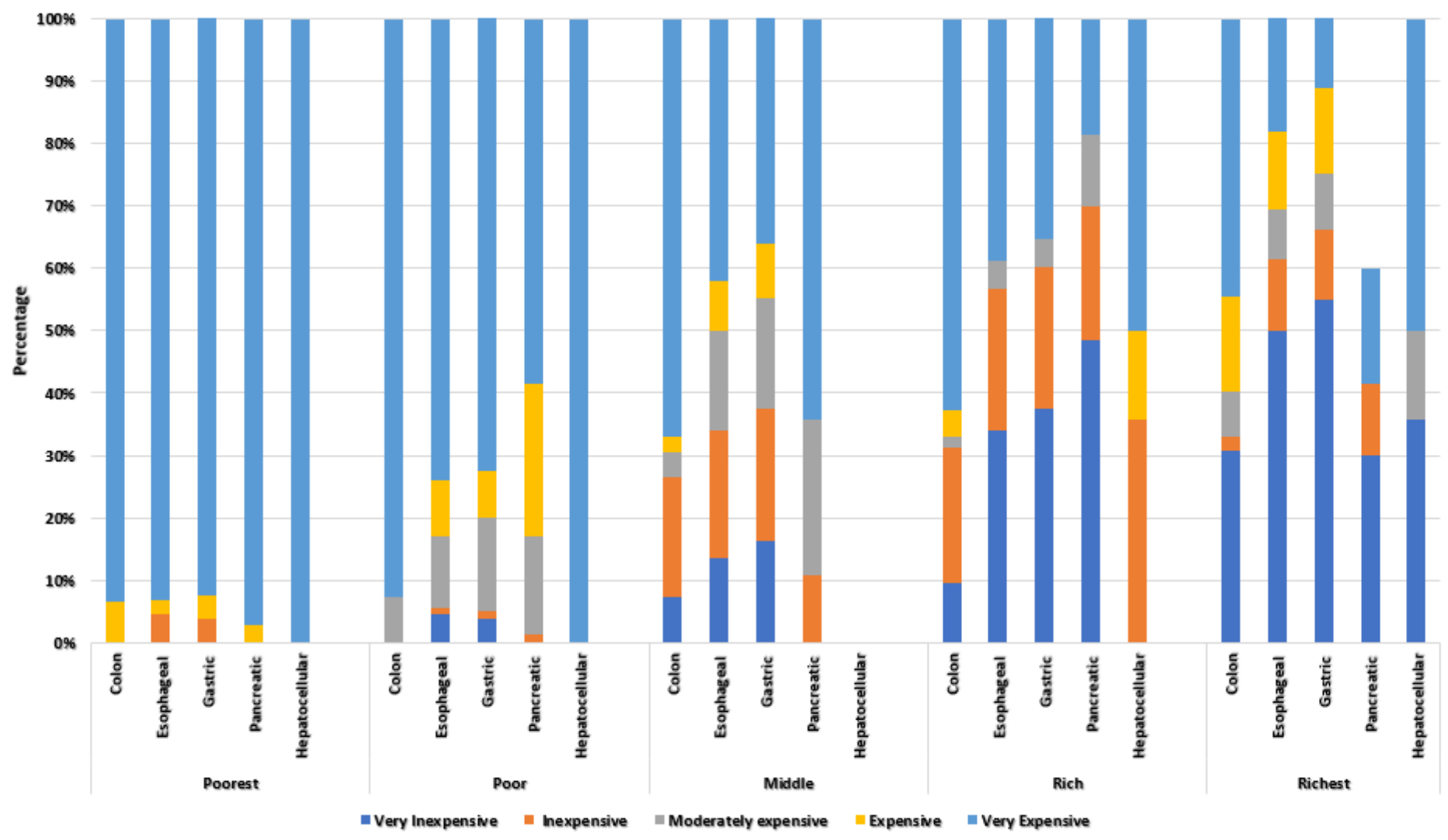

Figure 1: Affordability Grading by site of treatment and income class. 


\section{Affordability versus Efficacy of Treatment for Each Group of GIT Cancer Patients}

While poorest patient has no affordability for any treatment for GIT cancer, poor: n (\%) 7 (1.6), middle 49 (11.3), rich 110 (25.5) and richest has better affordability (Table 1 and Figure 2).

In colon cancer, 155 (93.4) of regimens are awfully expensive for poorest patient, 154 (92.8) for poor, 111 (66.9) for middle, 104 (62.7) for rich and 74 (44.6) for richest.

While esophageal cancer account for the only inexpensive regimens for poorest patient 4 (4.5), HCC account for the highest number of awfully expensive regimens for the richest group 14 (50).

There is significant negative relation between affordability and efficacy in middle, rich and income patient, which appear more in pancreatic cancer $\mathrm{P}<0.0001$ and appear also in richest HCC $\mathrm{P}<0.0001$.

\section{Affordability versus Safety of Treatment for Each Cancer Patient Group}

Most treatment regimens are not affordable for poorest and poor patients, but at the same time, there is positive week correlation between safety and affordability $\mathrm{P}<0.0001$, appear most in colon and gastric poor patients

For middle income patient with colon cancer, positive significant correlation occurs with $\mathrm{P}<0.0001$, while in HCC there is negative moderately strong correlation $\mathrm{P}=0.01$.

For rich group, the same correlation appears stronger and for richest patient it appears stronger $\mathrm{P}<0.0001$ (Figure 2).

\section{Results of the Use of Binary Logistic Regression Model for Relation between Affordability, Safety, and Efficacy}

Binary logistic regression model was performed to assess Affordability of cancer treatments in different cancer types using efficacy of regimen, safety of regimen, quality of evidence, consistency of evidence, line of therapy, site of treatment and income class as predictors (Table 3 and Figure 3).

The calculated quality measures showed good quality of fit for the established model were the $\mathrm{r}$ square $=0.550$ and Hosmer Lemeshow test revealed $\mathrm{p}=0.389$.

All predictors in the Model were clinically significant except quality of evidence and consistency of evidence.

Table 1: Affordability vs safety for each cancer treatment in the richest population.

\begin{tabular}{|c|c|c|c|c|c|c|}
\hline \multirow{2}{*}{ Site of treatment } & Efficacy & Highly toxic & Moderately toxic & Mildly toxic & Occasionally toxic & \multirow{2}{*}{$P$ value } \\
\hline & Affordability & $\mathrm{N}(\%)$ & $\mathrm{N}(\%)$ & $\mathrm{N}(\%)$ & $\mathrm{N}(\%)$ & \\
\hline \multirow{5}{*}{ Colon cancer } & Very inexpensive & $0(0 \%)$ & $5(8.6 \%)$ & $42(41.2 \%)$ & $4(100 \%)$ & \multirow{5}{*}{$\begin{array}{c}<0.0001^{¥} \\
\left(<0.0001^{€},\right. \\
\left.0.372^{\Sigma}\right)\end{array}$} \\
\hline & Inexpensive & $0(0 \%)$ & $3(5.2 \%)$ & $1(1 \%)$ & $0(0 \%)$ & \\
\hline & Moderately expensive & $0(0 \%)$ & $5(8.6 \%)$ & $7(6.9 \%)$ & $0(0 \%)$ & \\
\hline & Expensive & $0(0 \%)$ & $9(15.5 \%)$ & $16(15.7 \%)$ & $0(0 \%)$ & \\
\hline & Very Expensive & $2(100 \%)$ & $36(62.1 \%)$ & $36(35.3 \%)$ & $0(0 \%)$ & \\
\hline \multirow{5}{*}{$\begin{array}{l}\text { Esophageal and esophagogastric } \\
\text { junction cancer }\end{array}$} & Very inexpensive & $0(0 \%)$ & $16(55.2 \%)$ & $27(51.9 \%)$ & $1(14.3 \%)$ & \multirow{5}{*}{$\begin{array}{c}0.001^{¥} \\
\left(0.024^{€}\right. \\
\left.-0.240^{\Sigma}\right)\end{array}$} \\
\hline & Inexpensive & $0(0 \%)$ & $4(13.8 \%)$ & $6(11.5 \%)$ & $0(0 \%)$ & \\
\hline & Moderately expensive & $0(0 \%)$ & $2(6.9 \%)$ & $4(7.7 \%)$ & $1(14.3 \%)$ & \\
\hline & Expensive & $0(0 \%)$ & $7(24.1 \%)$ & $4(7.7 \%)$ & $0(0 \%)$ & \\
\hline & Very Expensive & $0(0 \%)$ & $0(0 \%)$ & $11(21.1 \%)$ & $5(71.4 \%)$ & \\
\hline \multirow{5}{*}{ Gastric cancer } & Very inexpensive & $0(0 \%)$ & $15(57.7 \%)$ & $26(53.1 \%)$ & $3(60 \%)$ & \multirow{5}{*}{$\begin{array}{c}0.115^{¥} \\
\left(0.546^{€},\right. \\
\left.-0.068^{\Sigma}\right)\end{array}$} \\
\hline & Inexpensive & $0(0 \%)$ & $4(15.4 \%)$ & $5(10.2 \%)$ & $0(0 \%)$ & \\
\hline & Moderately expensive & $0(0 \%)$ & $2(7.7 \%)$ & $3(6.1 \%)$ & $2(40 \%)$ & \\
\hline & Expensive & $0(0 \%)$ & $5(19.2 \%)$ & $6(12.2 \%)$ & $0(0 \%)$ & \\
\hline & Very Expensive & $0(0 \%)$ & $0(0 \%)$ & $9(18.4 \%)$ & $0(0 \%)$ & \\
\hline \multirow{5}{*}{ Pancreatic Adenocarcinoma } & Very inexpensive & $0(0 \%)$ & $1(12.5 \%)$ & $39(78 \%)$ & $0(0 \%)$ & \multirow{5}{*}{$\begin{array}{c}<0.0001^{¥} \\
\left(0.099^{€}\right. \\
\left.0.199^{\Sigma}\right)\end{array}$} \\
\hline & Inexpensive & $0(0 \%)$ & $7(87.5 \%)$ & $1(2 \%)$ & $4(33.3 \%)$ & \\
\hline & Moderately expensive & $0(0 \%)$ & $0(0 \%)$ & $0(0 \%)$ & $5(41.7 \%)$ & \\
\hline & Expensive & $0(0 \%)$ & $0(0 \%)$ & $0(0 \%)$ & $0(0 \%)$ & \\
\hline & Very Expensive & $0(0 \%)$ & $0(0 \%)$ & $10(20 \%)$ & $3(25 \%)$ & \\
\hline \multirow{5}{*}{ Hepatocellular carcinoma } & Very inexpensive & $0(0 \%)$ & $4(100 \%)$ & $6(25 \%)$ & $0(0 \%)$ & \multirow{5}{*}{$\begin{array}{c}0.015^{¥} \\
\left(0.007^{€},\right. \\
\left.-0.500^{\Sigma}\right)\end{array}$} \\
\hline & Inexpensive & $0(0 \%)$ & $0(0 \%)$ & $0(0 \%)$ & $0(0 \%)$ & \\
\hline & Moderately expensive & $0(0 \%)$ & $0(0 \%)$ & $4(16.7 \%)$ & $0(0 \%)$ & \\
\hline & Expensive & $0(0 \%)$ & $0(0 \%)$ & $0(0 \%)$ & $0(0 \%)$ & \\
\hline & Very Expensive & $0(0 \%)$ & $0(0 \%)$ & $14(58.3)$ & $0(0 \%)$ & \\
\hline
\end{tabular}


Rasha Aboelhassan (2022) Efficacy, Safety and Affordability Disparities of Gastrointestinal Cancer Changing Plans of Treatment of Cancer Patients

Table 2: relation between efficacy and safety for each cancer site

\begin{tabular}{|c|c|c|c|c|c|}
\hline \multirow{2}{*}{ Site of treatment } & Efficacy & Minimally effective & Moderately effective & Very effective & \multirow{2}{*}{ Correlation Coefficient ${ }^{1}$} \\
\hline & Safety & \multicolumn{3}{|c|}{ Number (\%) } & \\
\hline \multirow{4}{*}{ Colon cancer } & Highly toxic & $0(0)$ & $0(0)$ & $10(3.4)$ & \multirow{4}{*}{$(-0.091)^{\star *}$} \\
\hline & Moderately toxic & $40(34.8)$ & $140(32.9)$ & $110(37.9)$ & \\
\hline & Mildly toxic & $70(60.9)$ & $270(63.5)$ & $170(58.6)$ & \\
\hline & Occasionally toxic & $5(4.3)$ & $15(3.5)$ & $0(0)$ & \\
\hline \multirow{3}{*}{$\begin{array}{l}\text { Esophageal and } \\
\text { esophagogastric junction } \\
\text { cancer }\end{array}$} & Moderately toxic & $0(0)$ & $70(31.1)$ & $75(38.5)$ & \multirow{3}{*}{$(-0.149)^{* *}$} \\
\hline & Mildly toxic & $15(75)$ & $135(60)$ & $110(56.4)$ & \\
\hline & Occasionally toxic & $5(25)$ & $20(8.9)$ & $10(5.1)$ & \\
\hline \multirow{3}{*}{ Gastric cancer } & Moderately toxic & $5(20)$ & $80(44.4)$ & $45(23.1)$ & \multirow{3}{*}{$(0.028)$} \\
\hline & Mildly toxic & $5(20)$ & $90(50)$ & $150(76.9)$ & \\
\hline & Occasionally toxic & $15(60)$ & $10(5.6)$ & $0(0)$ & \\
\hline \multirow{3}{*}{ Pancreatic Adenocarcinoma } & Moderately toxic & $0(0)$ & $15(7.9)$ & $25(50)$ & \multirow{3}{*}{$(-0.359)^{\star *}$} \\
\hline & Mildly toxic & $75(68.2)$ & $160(84.2)$ & $15(30)$ & \\
\hline & Occasionally toxic & $35(31.8)$ & $15(7.9)$ & $10(20)$ & \\
\hline \multirow{2}{*}{ Hepatocellular carcinoma } & Moderately toxic & $20(66.7)$ & $0(0)$ & $0(0)$ & \multirow{2}{*}{$(0.653)^{\star *}$} \\
\hline & Mildly toxic & $10(33.3)$ & $95(100)$ & $15(100)$ & \\
\hline \multirow{4}{*}{ Total } & Highly toxic & $0(0)$ & $0(0)$ & $10(1.3)$ & \multirow{4}{*}{$(-0.152)^{* *}$} \\
\hline & Moderately toxic & $65(21.7)$ & $305(27.4)$ & $255(34.2)$ & \\
\hline & Mildly toxic & $175(58.3)$ & $750(67.3)$ & $460(61.7)$ & \\
\hline & Occasionally toxic & $60(20.0)$ & $60(5.4)$ & $20(2.7)$ & \\
\hline
\end{tabular}

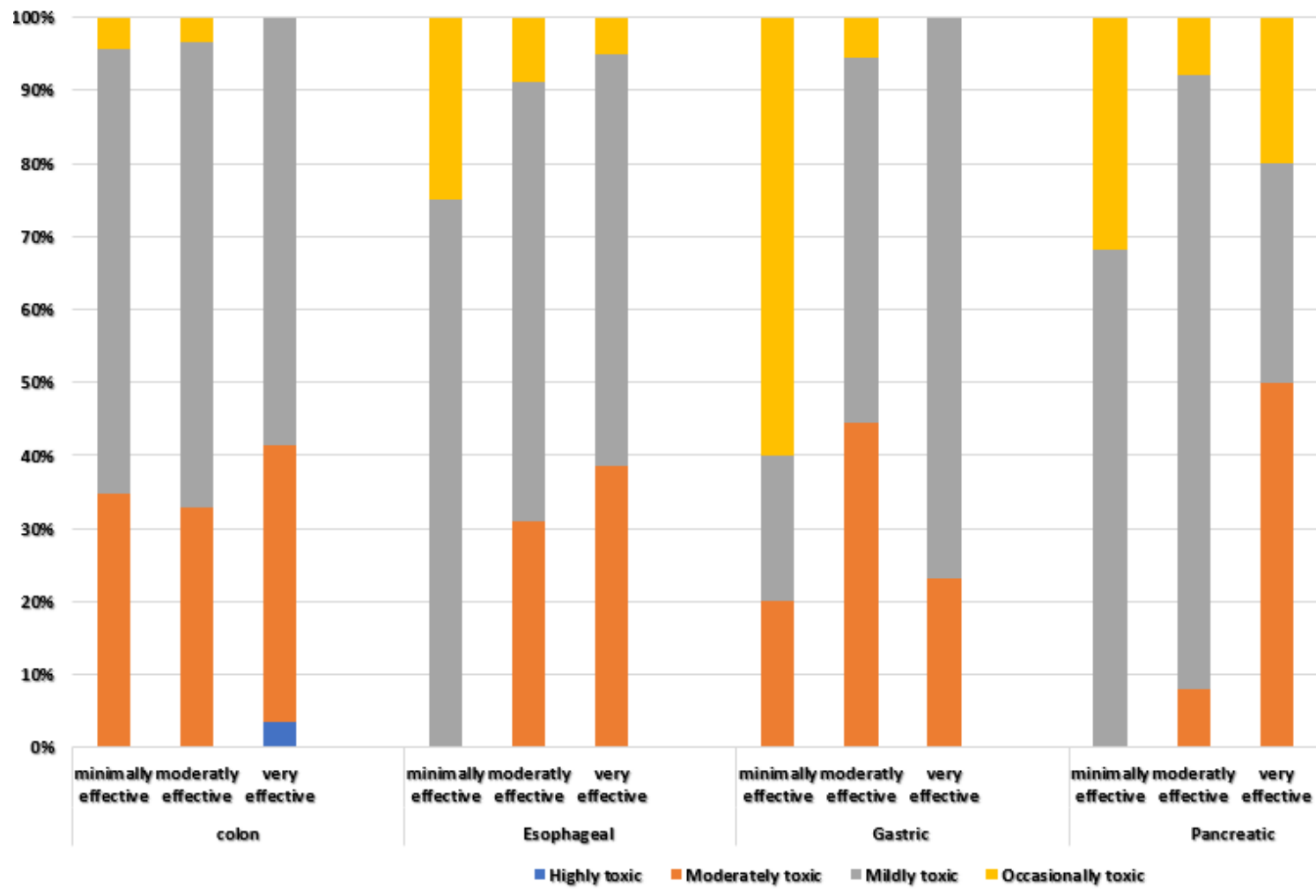

Figure 2: The relationship between efficacy of treatment and safety of treatment according to site of tumor. 
Rasha Aboelhassan (2022) Efficacy, Safety and Affordability Disparities of Gastrointestinal Cancer Changing Plans of Treatment of Cancer Patients

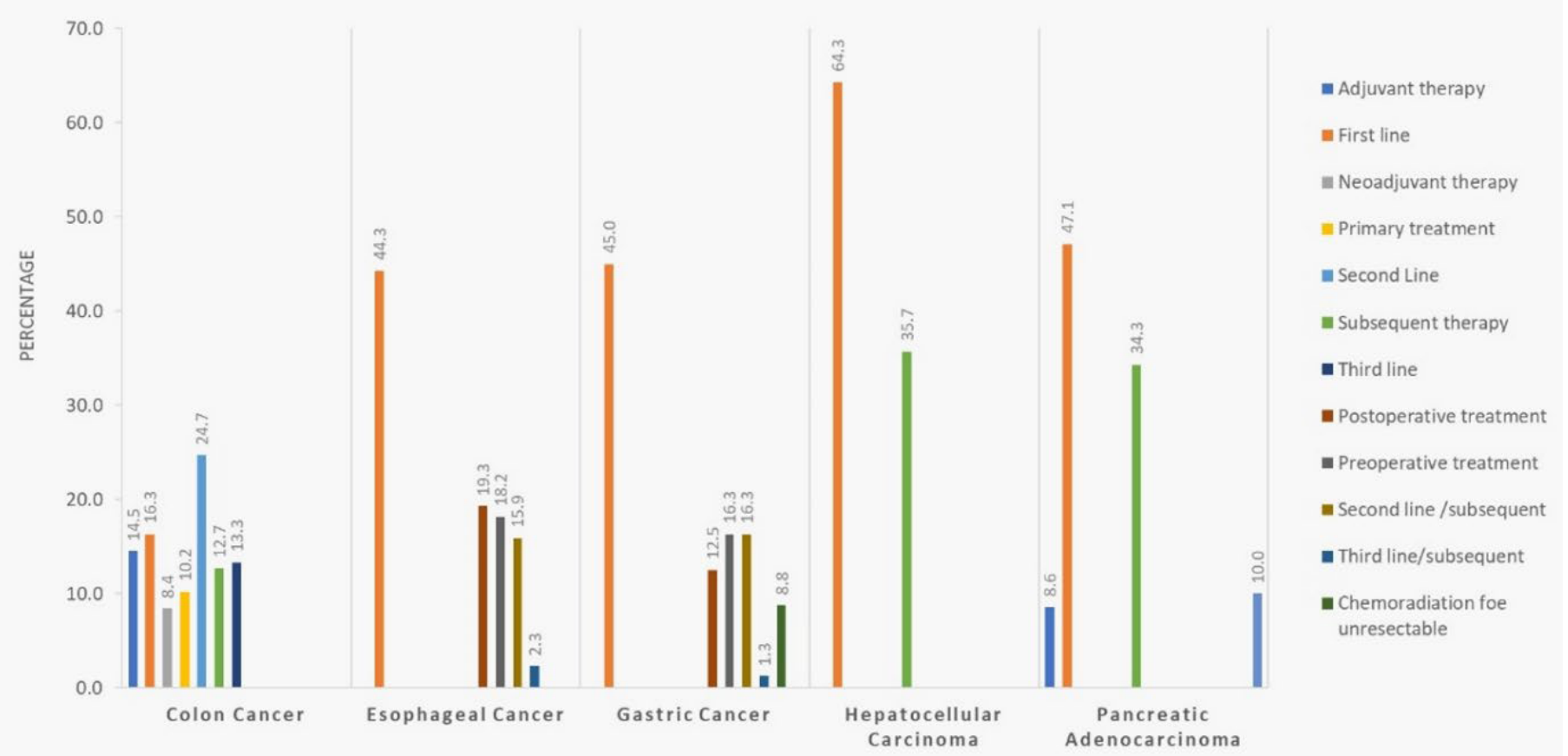

Figure 3: Percentage and distribution of lines of treatment by tumor sites:

Table 3: Binary logistic regression model measuring affordability relation with efficacy and safety for all GIT cancer sites

\begin{tabular}{|c|c|c|c|c|c|c|}
\hline Variables in the Equation & B & S.E. & Wald & $\mathrm{df}$ & Sig. & $\operatorname{Exp}(B)$ \\
\hline Efficacy of regimen & & & 75.318 & 2 & .000 & \\
\hline Minimally effective & 2.689 & .310 & 75.132 & 1 & .000 & 14.724 \\
\hline Moderately effective & 1.224 & .209 & 34.346 & 1 & .000 & 3.400 \\
\hline Safety of Regimen & & & 21.462 & 3 & .000 & \\
\hline High toxic & -17.885 & 11169.390 & .000 & 1 & .999 & .000 \\
\hline Moderately toxic & .226 & .339 & .443 & 1 & .506 & 1.253 \\
\hline Mildly Toxic & .961 & .311 & 9.525 & 1 & .002 & 2.613 \\
\hline Quality of evidence & & & 3.838 & 3 & .280 & \\
\hline Low quality & -.585 & 1.034 & .320 & 1 & .571 & .557 \\
\hline Average quality & -.086 & .950 & .008 & 1 & .927 & .917 \\
\hline Good quality & .278 & .928 & .090 & 1 & .764 & 1.321 \\
\hline Consistency of Evidence & & & 11.218 & 3 & .011 & \\
\hline Inconsistent & -17.824 & 4198.616 & .000 & 1 & .997 & .000 \\
\hline May be consistent & 1.186 & 1.386 & .732 & 1 & .392 & 3.274 \\
\hline Mainly consistent & 1.937 & 1.365 & 2.015 & 1 & .156 & 6.937 \\
\hline Income class & & & 256.165 & 3 & .000 & \\
\hline Middle & 3.025 & .422 & 51.455 & 1 & .000 & 20.589 \\
\hline Rich & 4.306 & .413 & 108.811 & 1 & .000 & 74.173 \\
\hline Richest & 5.620 & .419 & 179.873 & 1 & .000 & 275.753 \\
\hline Line of therapy & & & 140.645 & 2 & .000 & \\
\hline Neoadjuvant/Adjuvant & 2.858 & .246 & 135.048 & 1 & .000 & 17.428 \\
\hline Metastatic (first line) & 1.252 & .218 & 32.940 & 1 & .000 & 3.497 \\
\hline Site of treatment & & & 81.099 & 4 & .000 & \\
\hline Esophageal Cancer & .922 & .219 & 17.684 & 1 & .000 & 2.515 \\
\hline Gastric cancer & 1.317 & .226 & 33.938 & 1 & .000 & 3.733 \\
\hline Hepatocellular carcinoma & -.645 & .417 & 2.394 & 1 & .122 & .524 \\
\hline Pancreatic cancer & 1.825 & .244 & 56.109 & 1 & .000 & 6.202 \\
\hline Constant & -3.393 & 1.226 & 7.656 & 1 & .006 & .034 \\
\hline
\end{tabular}


The model showed that the minimally effective regimen has increased the chance of being affordable regimen by 15 times if compared to highly effective treatment $(\mathrm{OR}=14.724, \mathrm{P}<0.0001)$, while at the same time, the moderately effective drug increases the chance of affordability $\mathrm{t}$ by 3 times if compared to highly effective treatment $(\mathrm{OR}=3.40, \mathrm{P}<0.0001)$.

As for safety of regimen, the model shows that being a mildly toxic drug increase the chance of being affordable regimen by 3 times if compared to occasionally toxic regimen $(\mathrm{OR}=2.613, \mathrm{P}=0.002)$, however, highly toxic, and moderately toxic regimens did not show any difference in affordability if compared to occasionally toxic $(\mathrm{P}=0.999$ and $\mathrm{P}=0.506$ respectively).

As for line of therapy, the model shows that Neoadjuvant/Adjuvant regimens has increased the chance of being affordable treatment by 17 times if compared to Second line/subsequent treatment $(\mathrm{OR}=17.428$, $\mathrm{P}<0.0001$ ), while the first line treatment has increased chance of being affordable treatment by 3 times if compared to Second line/subsequent treatment $(\mathrm{OR}=3.497, \mathrm{P}<0.0001)$.

As for population income, patients from middle class increase the chance of being affordability for treatment by 20 times if compared to the poor/poorest class $(\mathrm{OR}=20.589, \mathrm{p}<0.0001)$, rich class increase the chance of being Affordable treatment by 74 times if compared to the poor/poorest class $(\mathrm{OR}=74.173, \mathrm{p}<0.0001)$, finally patients in richest class increase the chance of their affordability for treatment by more than 200 times if compared to the poor/poorest class $(\mathrm{OR}=275.753$, $\mathrm{p}<0.0001)$.

Finally, for site of treatment, the model shows that Esophageal cancer treatment regimens has increased chance of being affordable by 2 times if compared to Colon cancer $(\mathrm{OR}=2.515, \mathrm{P}<0.0001)$, while gastric cancer has increased chance of being affordable by 3 times if compared to Colon cancer $(\mathrm{OR}=3.733, \mathrm{P}<0.0001)$, Pancreatic cancer has the increased chance of being affordable by 6 times if compared to Colon cancer $(\mathrm{OR}=6.202, \mathrm{P}<0.0001)$, however hepatocellular carcinoma did not show any difference in affordability if compared to colon cancer.

\section{The Results of Relation between Efficacy and Safety}

In total sample, the relationship between efficacy and safety is negative and weak, $(-0.152$, $\mathrm{p}$-value $<0.01)$, which means that as the treatment becomes more effective, it is supposed to become less toxic but in weak manner as the value of correlation coefficient is 0.152 less than 0.3 which is the cutoff point of weak correlation (Table 1 and Figure 2).

For colon cancer, esophageal and esophagogastric junction cancer, the correlation between safety and efficacy is negative and weak, ( -0.091 and -0.149 respectively), but for Pancreatic Adenocarcinoma, the correlation is negative moderate (-0.359). On the contrary, only for Hepatocellular carcinoma, the correlation between safety and efficacy is positive and strong (0.653).

\section{Discussion}

Our study confirmed that not all economy levels of patients with GIT cancer can afford for effective safe treatment, raising the needs for insurance and help from sponsors in most levels of social classes, at the same time, there is significant negative relation between efficacy and affordability for GIT cancer therapy.

Our explanation for this relation is that, when we need to increase efficacy for cancer therapy, we add another chemotherapy, which dose not only add to treatment price and efficacy, but it also decreases safety of cancer treatment.

Another way for increase efficacy is to add immunotherapy or targeted therapy, which are both expensive for most of patient income groups in the study.

Our study also concluded that treatment of early stages of cancer are more affordable than late stages, which will encourage governments to add more efforts for early detection of GIT cancer which will not only save lives but also will save money.

Treatment of some cancer sites like esophagus and stomach are more affordable than others like colon, this may be because investment in common cancers like colon cancer are more common, which increase price of treatment these cancers.

For poor and poorest patients, our recommendation is to do more screening for early detection of cancer in these groups and on the other hand, using bio similar treatment with lower prices may be of benefit.

According to recent salary survey [9], more than $75 \%$ of Egyptian population needs support from insurance for GIT cancer therapy as their monthly income is less than 44000 EGP, which directed governmental plans to invest more in screening and early detection of cancer.

Considering the common GIT cancer in Egypt like HCC, which has the least affordability for richest population in advanced stages according to our results, detection in early stages will be having greatest benefit for survival, more than late stages.

According to our results, relation between efficacy and safety is positive and strong for HCC, very effective regimens and moderately effective regimens are mildly toxic, while minimally effective regimens are moderately toxic, most of these lines of systemic therapy are for first line advanced disease, with low survival benefit.

\section{References}

1. Arnold, Melina, Christian C. Abnet, Rachel E. Neale, Jerome Vignat, Edward L. Giovannucci, et al. (2020) Global burden of 5 major types of gastrointestinal cancer. Gastroenterology 159: 335-349. [crossref]

2. Cortes, Javier, Jose Manuel Perez-García, Antonio Llombart-Cussac, Giuseppe Curigliano, et al. (2020) Enhancing global access to cancer medicines. CA: a cancer journal for clinicians 70: 105-124. [crossref]

3. Shulman, Lawrence N., Claire M. Wagner, Ronald Barr, et al. (2016) Proposing essential medicines to treat cancer: methodologies, processes, and outcomes. Journal of Clinical Oncology 34: 69. [crossref]

4. Aggarwal A, Fojo T, Chamberlain C, Davis C, Sullivan R (2017) Do patient access schemes for high-cost cancer drugs deliver value to society? Lessons from the NHS Cancer Drugs Fund. Ann Oncol 28:1738-1750. [crossref]

5. Cherny, Nathan I., Richard Sullivan, Urania Dafni, J. Martijn Kerst, et al. (2015) A standardised, generic, validated approach to stratify the magnitude of clinical benefit that can be anticipated from anti-cancer therapies: the European Society for Medical Oncology Magnitude of Clinical Benefit Scale (ESMO-MCBS). Annals of Oncology 26: $1547-1573$. 
Rasha Aboelhassan (2022) Efficacy, Safety and Affordability Disparities of Gastrointestinal Cancer Changing Plans of Treatment of Cancer Patients

6. Shah-Manek, Bijal, Joseph S. Galanto, Huong Nguyen, and Robert Ignoffo (2017) Value frameworks for the patient-provider interaction: a comparison of the ASCO value framework versus NCCN evidence blocks in determining value in oncology. Journal of Managed Care \& Specialty Pharmacy 23: S13-S20.

7. Shah-Manek, Bijal, William Wong, Arliene Ravelo, and Marco DiBonaventura (2018) Oncologists' perceptions of drug affordability using NCCN evidence blocks results from a national survey. Journal of Managed Care \& Specialty Pharmacy 24: 565-571.
8. Salas-Vega, Sebastian, Emily Shearer, and Elias Mossialos (2020) Relationship between costs and clinical benefits of new cancer medicines in Australia, France, the UK, and the US. Social Science \& Medicine 258: 113042. [crossref]

9. http://www.salaryexplorer.com/salary-survey.php?loc=64\&loctype=1\#disabled

10. Khatib R, McKee M, Shannon H, Chow C, Rangarajan S, et al. (2016) Availability and affordability of cardiovascular disease medicines and their effect on use in highincome, middle-income, and low-income countries: an analysis of the PURE study data. Lancet 2;387(10013): 61-9. [crossref]

\section{Citation:}

Aboelhassan R, Ali S, Ali MH, Abdel-Fatah NA (2022) Efficacy, Safety and Affordability Disparities of Gastrointestinal Cancer Changing Plans of Treatment of Cancer Patients. Cancer Stud Ther J Volume 7(1): 1-7. 